\title{
Influence of particulate matter air pollution on exacerbation of chronic obstructive pulmonary disease depending on aerodynamic diameter and the time of exposure in the selected population with coexistent cardiovascular diseases
}

The authors declare no financial disclosure

\begin{abstract}
Introduction: Particulate matter air pollution constitutes an important factor affecting the course of various respiratory and cardiovascular diseases. Two main monitored groups of particulate pollution are particulate matter with the aerodynamic diameter below $10 \mu \mathrm{m}$ (PM10) and $2.5 \mu \mathrm{m}$ (PM2.5). One of the most important respiratory diseases is chronic obstructive pulmonary disease (COPD). Clinical presentation of COPD and cardiovascular diseases is similar and can cause complications during therapy. The study explores connection between particulate matter and COPD exacerbations in population with cardiovascular cause of symptoms excluded.

Material and methods: Analysis was based on data from hospitalisations in the years 2006-2016 in the hospitals of Upper Silesian Agglomeration, Poland. The data were correlated with meteorological conditions and particulate matter concentrations up to 90 days before hospital admission.

Results: During the whole observation period no connection between PM10 concentration changes and COPD exacerbations were observed. On the other hand PM 2,5 influence started to be significant on 14 day before admission (RR 1.06) and increased up to maximal analysed period of 90 days (RR 1.32).

Conclusions: Overall this study highlights the importance of particulate matter pollution emission impact on COPD exacerbations in a long time perspective.
\end{abstract}

Key words: air pollution, particulate matter, COPD exacerbations, cardiovascular comorbidities

Adv Respir Med. 2018; 86: 227-233

\section{Introduction}

Available literature concentrates on the effect of air pollution on the prevalence of exacerbation episodes of respiratory and cardiovascular diseases. The evidence about exacerbation of respiratory diseases, including chronic obstructive pulmonary disease (COPD) in the population of patients suffering from cardiovascular diseases, is scarce.
Particular matter constitutes a mixture of chemical compounds, heterogenous in terms of composition and physical properties, differing depending on their source. The World Health Organisation (WHO) points to the increase of the incidence rate of adverse effects of exposure to PM along with the growth of the concentrations thereof. At the same time it is difficult to determine the minimum concentration below which

Address for correspondence: Michał Zieliński, Department of Lung Diseases and Tuberculosis, School of Medicine with the Division of Dentistry in Zabrze,

Medical University of Silesia in Katowice, Poland, e-mail: michal.zielinski1@interia.pl

DOI: 10.5603/ARM.2018.0036

Received: 29.07 .2018

Copyright (C) 2018 PTChP

ISSN 2451-4934 
such effects do not occur [1]. In 2010, PM contributed to over 3 million deaths, which constitutes an increase by 300 thousand in relation to 1990 . $\mathrm{PM}$ is a significant factor exacerbating the course of respiratory and cardiovascular diseases [2]. Furthermore, a growing body of evidence links PM to COPD development, which led to the inclusion of $\mathrm{PM}$ in a group of important factors contributing to COPD prevalence in the annual GOLD report [3].

In 2002, COPD was the fifth leading cause of death worldwide, and is projected to be the third by 2020 [4]. The natural course of the disease features exacerbation periods, which accelerate the drop in lung function tests values, contribute to the mortality rate increase, and significantly elevate healthcare systems costs [4-5]. The main cause of exacerbation in COPD are viral and bacterial infections. Ambient pollution and meteorological factors are also considered among precipitating agents or cofactors [4]. It has been demonstrated that there is a relation between exposure to PM and intensification of local inflammation in the area of the respiratory tract, as well as a systemic inflammatory response in consequence of changes in phagocytosis, production of chemotactic compounds, cytokines and oxygen free radicals [6]. It has been proven that there is a relation between exposure to PM, the drop of spirometric values [7-8], increase of dyspnoea, wheezes in patients with asthma and COPD [9]. It entailed higher consumption of inhalation medications applied in the treatment of COPD [10] and a bigger number of hospitalisations due to COPD exacerbation [11-13]. Simultaneously, the fundamental symptom of the COPD exacerbation - intensified dyspnoea above the normal variability - occurs in the course of exacerbation of congestive heart failure (CHF) and other cardiovascular diseases. CHF patients can present obstructive ventilation disorders too, which may be suggestive of the comorbidity or presence of COPD. Owing to similar risk factors (smoking), the population of cardiovascular patients constitutes a group predisposed to the development of COPD. A similar clinical picture, as well as the frequent comorbidity of cardiovascular diseases and COPD, account for the diagnostic and therapeutic challenge in the event of exacerbation. Moreover, it introduces potential bias in studies analysing PM concentrations and natural course of those diseases [14].

This study analyses the effect of PM with the aerodynamic diameter below $10 \mu \mathrm{m}$ (PM10) and $2.5 \mu \mathrm{m}$ (PM2.5) on COPD exacerbation in the population of cardiovascular patients depending on the exposure time. In order to avoid an erroneous classification of patients symptoms, cardiovascular database was employed. The aim of the study was the selection of patients with stable cardiovascular disease and concomitant COPD exacerbation, thus excluding the possibility of misdiagnosing the source of patients complaints.

\section{Material and methods}

The Silesian Cardiovascular Database (Ślqska Baza Sercowo-Naczyniowa) served as a source of data concerning hospitalisations due to COPD exacerbation. This database is administered by the Science Department of Silesian Centre of Heart Disease in Zabrze and the Department of Biostatistics of the Medical University of Silesia in Katowice under an agreement signed with the Silesian Provincial Department of the Polish National Health Fund. It contains information relating to hospitalisations in the wards of cardiology, cardiac surgery, vascular surgery, diabetology, internal medicine, and intensive care units with diagnosed conditions classified as cardiovascular diseases (coded as I01-I99 according to the International Classification of Diseases ICD-10). It covers hospitalisations in the years 2006-2016 in 310 hospitals. The inclusion criterion for patients was COPD exacerbation defined as the primary hospitalisation diagnosis coded as J44 according to ICD-10. Cardiovascular diseases were ruled out as the cause of symptoms reported by the patient. It corresponded to 12,889 hospitalisations, whose data are included in the database.

The data relating to particular matter pollution, i.e. PM2.5 and PM10, were obtained from the Upper Silesian air quality monitoring system database available for the public on the website of the Silesian Regional Inspectorate of Environment. Furthermore, this register provided information on meteorological conditions - ambient temperature, humidity, wind velocity, atmospheric pressure. The analysis covered figures relating to air monitoring stations located in Dąbrowa Górnicza, Gliwice, Katowice, Sosnowiec, Tychy, and Zabrze, adequately to the analysed population of the Upper Silesian Agglomeration. The Upper Silesian Agglomeration is a part of Silesian Voivodeship which constitutes $4 \%$ of Poland surface with $18.1 \%$ of industrial factories. Regional industry emits $23 \%$ of the whole country particulate air pollution.

Statistical analysis was performed using the generalised linear model with the logarithmic link 
Table 1. The most frequent cardiovascular comorbidities

\begin{tabular}{lllcc}
\hline No. & ICD-10 & Comorbidity & Number of patients & Percentage \\
\hline 1 & I10 & Essential (primary) hypertension & 3461 & 26.85 \\
2 & 150.9 & Heart failure, unspecified & 1370 & 10.63 \\
3 & 125.9 & Chronic ischaemic heart disease, unspecified & 1334 & 10.35 \\
4 & 125.0 & Atherosclerotic cardiovascular disease, so described & 1038 & 8.05 \\
5 & 148 & Atrial fibrillation and flutter & 906 & 7.03 \\
6 & 111.9 & Hypertensive heart disease without (congestive) heart failure & 901 & 6.99 \\
7 & 170.9 & Generalised and unspecified atherosclerosis & 863 & 6.7 \\
8 & 150.0 & Congestive heart failure & 644 & 5 \\
9 & 125.8 & Other forms of chronic ischaemic heart disease & 470 & 3.65 \\
10 & I25.5 & Ischaemic cardiomyopathy & 397 & 3.08 \\
\hline
\end{tabular}

ICD-10: International Classification of Diseases

Table 2. Mean, standard deviation (SD) and range of yearly concentrations of PM pollution

\begin{tabular}{ccccccccc}
\hline Year & $\mathbf{P M}_{\mathbf{1 0}}\left[\boldsymbol{\mu \mathbf { g }} / \mathbf{m}^{3}\right]$ & $\mathbf{S D}$ & $\mathbf{M I N}$ & $\mathbf{M A X}$ & $\mathbf{P M}_{\mathbf{2 . 5}}\left[\boldsymbol{\mu g} / \mathbf{m}^{3}\right]$ & $\mathbf{S D}$ & MIN & MAX \\
\hline 2006 & 50.16 & 45.89 & 8.67 & 385.75 & unavailable & - & - & - \\
2007 & 39.27 & 25.05 & 10.75 & 185.2 & unavailable & - & - & - \\
2008 & 40.07 & 23.15 & 10.75 & 169.75 & unavailable & - & - & - \\
2009 & 41.07 & 25.56 & 8.25 & 159.75 & 36.13 & 23.84 & 6 & 140.5 \\
2010 & 53.19 & 41.08 & 7.33 & 254.6 & 45.92 & 42.96 & 6 & 313 \\
2011 & 54.69 & 40.16 & 9.5 & 356.33 & 42.39 & 35.05 & 5 & 223 \\
2012 & 52.81 & 48.33 & 9.8 & 410.17 & 40.75 & 46.07 & 5.5 & 358.5 \\
2013 & 43.36 & 24.47 & 12.17 & 192.67 & 35.42 & 27.23 & 4. & 205 \\
2014 & 41.68 & 26.61 & 9.67 & $163 / 5$ & 35.02 & 28.88 & 3.5 & 147.5 \\
\hline
\end{tabular}

function. Besides the season of the year, the following meteorological parameters were assumed as interfering variables: ambient temperature, humidity, wind velocity, atmospheric pressure. The Poisson distribution of the dependent variable was assumed. Measurements from the monitoring stations referring to the whole day were averaged for the Upper Silesian Agglomeration and analysed in juxtaposition with the hospitalisation data for the basic diagnosis J44. The period of up to 90 days before the day of hospital admission was considered. Statistical significance was assumed for $\mathrm{p}<0.05$. Relative risk was calculated for 1 interquartile range increase in the pollutant concentration.

\section{The analysed population}

From amongst hospitalisations subjected to the analysis, $61.82 \%$ were male patients, and $38.18 \%$ were females. The average age as of the time of admission was 71 years with the standard deviation of 10 years. The whole population of the Upper Silesian Agglomeration is estimated at up to 3.5 million.

\section{Cardiovascular comorbidities}

Besides the main diagnosis, hospitalisation reporting to the Polish National Health Fund contains up to three diagnoses of comorbidities. Table 1 provides information on 10 most frequent cardiovascular diagnoses in the analysed population.

\section{Results}

\section{PM concentrations in the analysed period}

PM10 measurements were available for the entire period analysed. Values of PM2.5 concentrations were available from June 2009 until the end of the analysed period. The mean yearly concentrations of PM pollution are collected in Table 2. The weekly concentrations of particulate pollutants for an exemplary year are presented in Figure 1. 


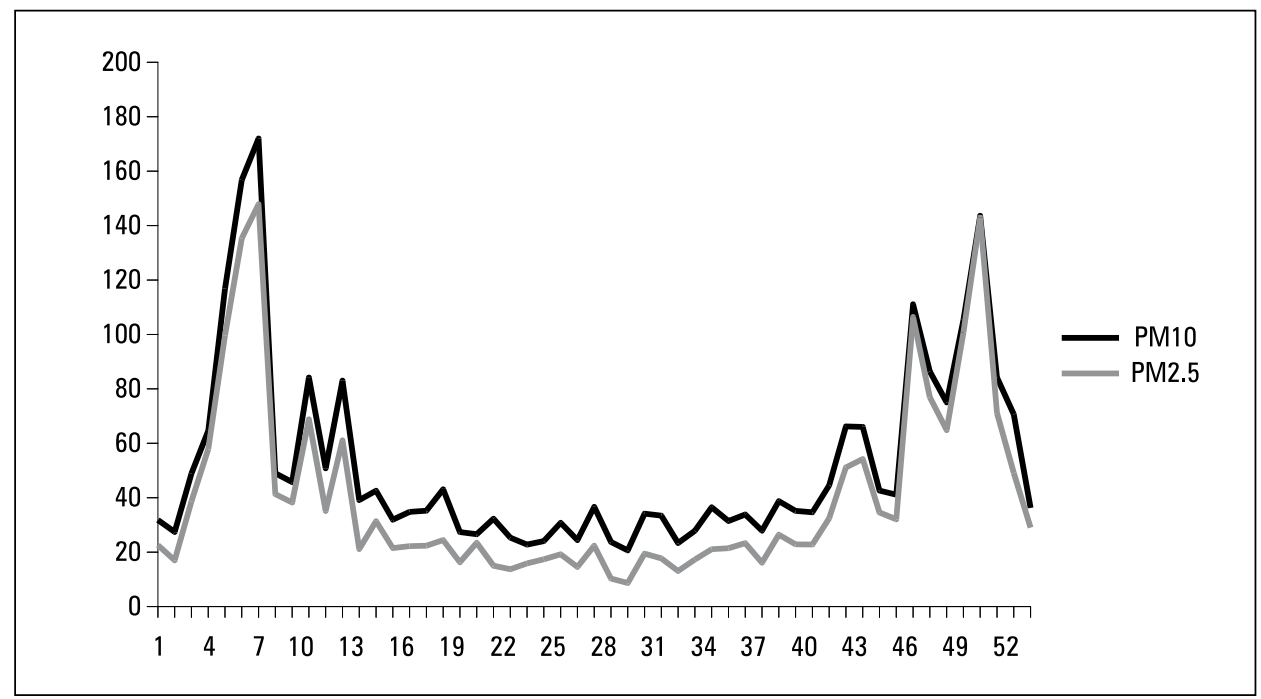

Figure 1. Plot of the weekly PM10 and PM2.5 densities over the year 2012

\section{Impact of PM concentrations on COPD exacerbation}

No significant relationship between a change in the PM10 concentration up to the 90th day before hospitalisation and the risk of COPD exacerbation was demonstrated in the analysed population. A relation between a change in the PM2.5 concentration and exacerbation was shown for the mean concentration for the period of over 14 days before the date of admission. The relative risk value (RR) was calculated per 1 interquartile range of pollutant concentration increase, equalling $29.58 \mu \mathrm{g} / \mathrm{m}^{3}$ for PM10 and $25.00 \mu \mathrm{g} / \mathrm{m}^{3}$ for PM2.5.

\section{Discussion and conclusions}

To the best of our knowledge, this paper is the first to focus on analysis of effects of PM pollutants on COPD exacerbation in patients with cardiovascular comorbidities.

The results obtained point to a significant effect of an increase in the PM2.5 concentration, but not PM10 concentration, on COPD exacerbation in the period of at least 2 weeks before the date of admission to hospital.

Standard concentrations of PM pollutants quoted by WHO, besides target values, provide also transitional ones. Mean annual levels in the analysed period did not go beyond $55 \mu \mathrm{g} / \mathrm{m}^{3}$ for PM10; for PM2.5 mean concentrations were below $46 \mu \mathrm{g} / \mathrm{m}^{3}$. The values obtained by us point to reaching the second transitional value proposed by WHO in terms of PM10, and the first transitional value in terms of PM2.5. These levels correspond to the $9 \%$ and $15 \%$ increase, respectively, in the risk of long-term mortality rate compared to the target values [1]. International guidelines indicate a significant role of pollutants in the pathogenesis of cardiovascular and respiratory diseases, even in concentrations remaining below the actual air quality standards [15].

In a study covering the area of Beijing, where the observed concentrations of PM are ca. two or three times higher than in our study, an increase in the intensity of inflammation and a rise of the number of reported respiratory symptoms was observed. In contrast to our results, changes in the concentrations of PM2.5 and PM10 within the range of up to 7 days had a significant effect [16]. A 6-month-long observation from European research centres pointed to a relationship between PM10 and respiratory symptoms. Similar connection for PM2.5 was not found. The authors emphasise a bigger role of PM10 in the toxicity of PM pollution. Nevertheless, whereas the demonstrated values of concentrations of PM10 are comparable with the ones determined in our study, the observed concentrations of PM2.5 are significantly lower [17]. An analysis based on the population of Massachusetts, USA, where the observed concentrations of PM pollutants fall within the range of target concentrations according to WHO, demonstrated the odds ratio (OR) of 0.94 at the $95 \%$ confidence interval (95\%CI) $0.88-1.01$ for COPD exacerbation with the exposure to PM2.5. This value took into account ambient temperature and reported infections of the respiratory tract as important confounders. The analysis covered a 3- week-long period, and considering the confidence interval given in the study, the 
Table 3. Impact of PM pollution concentration increase on the risk of chronic obstructive pulmonary disease exacerbation requiring inpatient treatment. Statistically significant associations are shown in bold

\begin{tabular}{|c|c|c|c|c|}
\hline \multirow[b]{2}{*}{ Analysed period } & \multicolumn{2}{|c|}{ PM10 } & \multicolumn{2}{|c|}{ PM2.5 } \\
\hline & $\mathbf{R R}$ & $\mathbf{p}$ & $\mathbf{R R}$ & $\mathbf{p}$ \\
\hline At admission & 1.02 & 0.11 & 1.00 & 0.79 \\
\hline last 3 days before admission & 1.00 & 0.71 & 1.00 & 0.89 \\
\hline last 5 days before admission & 0.99 & 0.70 & 1.01 & 0.71 \\
\hline last 7 days before admission & 1.00 & 0.95 & 1.02 & 0.33 \\
\hline last 14 days before admission & 1.01 & 0.50 & 1.06 & $<0.05$ \\
\hline last 30 days before admission & 1.01 & 0.81 & 1.10 & $<0.005$ \\
\hline last 40 days before admission & 0.99 & 0.79 & 1.14 & $<0.0005$ \\
\hline last 50 days before admission & 1.01 & 0.79 & 1.21 & $<0.0001$ \\
\hline last 60 days before admission & 1.01 & 0.81 & 1.24 & $<0.0001$ \\
\hline last 90 days before admission & 1.00 & 0.85 & 1.32 & $<0.0001$ \\
\hline
\end{tabular}

RR: relative risk; $p$ : probability value

obtained results also point to the lack of effect for a short exposure time, as demonstrated by us [18]. A meta-analysis examining the relationship between air pollutants and hospital admissions due to COPD exacerbation revealed a marginal relationship (OR 1.01, 95\%CI 1.0-1.01) for PM10. The influence of PM2.5 was likewise marginal, although expressed more profoundly (OR 1.03, 95\%CI 1.01-1.05). For the sake of comparison, the results obtained by us point to a much higher $\mathrm{RR}$, in the time perspective of 90 days obtaining the value 1.32. The authors of the meta-analysis underline that the relationship between the concentration of PM2.5 and COPD exacerbation becomes more distinct for higher concentrations of PM2.5 [19]. De Vries et al. in their work empasise the significance of a delay in exerting the effect due to pollution and, consequently, of the need to choose an appropriate period for the analysis. For PM2.5, the strongest effect is marked when a mean value covering several preceding days is applied. It is justified by experimental studies pointing to a cumulative role of modification of elements of the immunological reaction, such as chemotaxis and phagocytosis [20]. Furthermore, when comparing data obtained in different studies, one needs to take into account the difference in the composition of PM pollution, resulting from different sources and secondary chemical reactions. A study contrasting the influence of PM10 in 29 European cities demonstrated significant heterogeneity depending on the observed area [21]. Similar works pertaining to the effect of PM2.5 pointed to increased mortality related to PM2.5 generated by human activity, which is the main source of air pollution in the area covered by our study [22, 23].

The data obtained by us are based on information from hospitalisation reporting. Therefore, they disregard the effect of PM pollution on the cases of exacerbation which do not require hospitalisation, or patients who do not consent to the inpatient treatment. Studies focusing on the sales of respiratory medication demonstrated a relationship between the concentration of PM10 and the sales of medications used in the treatment of asthma and COPD. The effect of PM manifested itself on the date of the drug purchase, as well as for a mean value for various lag periods. Nevertheless, the analysed population potentially covered also patients with asthma and other respiratory diseases $[24,25]$. Data pertaining to the increase in the number of outpatient consultations resulting from the increased exposure to PM pollution are poorer than the data concerning hospitalisations. The study based on the observations in Beijing points to a very insignificant relation, although it covers only one centre, which may result in an analysis of a non-representative sample [26]. An analogous research covering an industrialised territory of Taiwan demonstrated a positive correlation between PM2.5 and PM10 concentrations on one hand and outpatient visits caused by COPD on the other [27]. The above mentioned studies indicate the possibility of underestimation of the effect of PM pollution on COPD exacerbation if only hospitalisations are taken into consideration.

The statistical analysis took into account variables relating to meteorological phenomena as 
disturbing variables. It was demonstrated that an increase in the air temperature outside buildings is connected with a rise of respiratory symptoms, such as dyspnoea, coughing, and expectoration [28]. Temperature increase by $1^{\circ} \mathrm{C}$ entails an increase of the incidence of COPD exacerbation estimated at the level of $2.7-5.4 \%$, depending on the study. In one of the studies, it translated into an increase of emergency admissions to hospital [29, 30]. Other meteorological factors were subjected to a less comprehensive analysis. A correlation between the number of outpatient consultations due to COPD and the atmospheric pressure, wind velocity, humidity, and insolation was presented, although it proved impossible to demonstrate this relationship for some parts of the analysed area [31, 32]. Furthermore, meteorological variables influence the concentration of PM pollution affecting a lag between concentration change and clinical manifestation [33].

In our study, we assumed a mean value of pollutants concentrations as the exposure measure. Depending on the amount of time spent inside buildings, the use of air conditioning or even a small change in environment, these values might not correspond to the actual exposure of a specific individual $[34,35]$. The exposition differences and subsequent clinical changes were demonstrated for two locations with various characteristic in London [36]. Similar differences were proven for coastal regions and areas without contact with seashore [37].

Basing the analysis on the cardiovascular diseases database allowed to limit the risk of inappropriate classification of the source of health issues of the patient. At the same time, it led to the analysis being performed on a selected population with different characteristics than those of the general population. Several causes of hospitalisation cannot be ruled out, either. Regrettably, the data reporting system used by the National Health Fund does not contain information on present smoking status. A number of studies suggest different pollutants impact depending on the actual smoking habit [38].

\section{Conflict of interest}

The authors declare no conflict of interest.

\section{References:}

1. Air quality guidelines. Global update 2005. Particulate matter, ozone, nitrogen dioxide and sulfur dioxide. WHO Press, Geneva, 2005.

2. Lim SS, Vos T, Flaxman AD, et al. A comparative risk assessment of burden of disease and injury attributable to 67 risk factors and risk factor clusters in 21 regions, 1990-2010: a systematic analysis for the Global Burden of Disease Study 2010. Lancet. 2012; 380(9859): 2224-2260, doi: 10.1016/ S0140-6736(12)61766-8, indexed in Pubmed: 23245609.

3. Global Strategy for the Diagnosis, Management, and Prevention of Chronic Obstructive Pulmonary Disease. 2018 Report. https://goldcopd.org; 30.09.2018.

4. Ko FW, Chan KaP, Hui DS, et al. Acute exacerbation of COPD. Respirology. 2016; 21(7): 1152-1165, doi: 10.1111/resp.12780, indexed in Pubmed: 27028990.

5. Donaldson GC, Seemungal TAR, Bhowmik A, et al. Relationship between exacerbation frequency and lung function decline in chronic obstructive pulmonary disease. Thorax. 2002; 57(10): 847-852, indexed in Pubmed: 12324669.

6. Ni L, Chuang CC, Zuo Li. Fine particulate matter in acute exacerbation of COPD. Front Physiol. 2015; 6: 294, doi: 10.3389/ fphys.2015.00294, indexed in Pubmed: 26557095.

7. Forbes LJL, Kapetanakis V, Rudnicka AR, et al. Chronic exposure to outdoor air pollution and lung function in adults. Thorax. 2009; 64(8): 657-663, doi: 10.1136/thx.2008.109389, indexed in Pubmed: 19359266.

8. Guo C, Zhang Z, Lau AKH, et al. Effect of long-term exposure to fine particulate matter on lung function decline and risk of chronic obstructive pulmonary disease in Taiwan: a longitudinal, cohort study. Lancet Planet Health. 2018; 2(3): e114-e125, doi: 10.1016/S2542-5196(18)30028-7, indexed in Pubmed: 29615226.

9. Karakatsani A, Analitis A, Perifanou D, et al. Particulate matter air pollution and respiratory symptoms in individuals having either asthma or chronic obstructive pulmonary disease: a European multicentre panel study. Environ Health. 2012; 11: 75, doi: 10.1186/1476-069X-11-75, indexed in Pubmed: 23039312.

10. Casas L, Simons K, Nawrot TS, et al. Respiratory medication sales and urban air pollution in Brussels (2005 to 2011). Environ Int. 2016; 94: 576-582, doi: 10.1016/j.envint.2016.06.019, indexed in Pubmed: 27346740.

11. Tamayo-Uria I, Altzibar JM, Mughini-Gras L, et al. Exacerbations of Chronic Obstructive Pulmonary Disease (COPD): An Ecological Study in the Basque Country, Spain (2000-2011). COPD. 2016; 13(6): 726-733, doi: 10.1080/15412555.2016.1182145, indexed in Pubmed: 27232203.

12. Faustini A, Stafoggia M, Colais P, et al. EpiAir Collaborative Group. Air pollution and multiple acute respiratory outcomes. Eur Respir J. 2013; 42(2): 304-313, doi: 10.1183/09031936.00128712, indexed in Pubmed: 23314899.

13. Liu Y, Yan S, Poh K, et al. Impact of air quality guidelines on COPD sufferers. Int J Chron Obstruct Pulmon Dis. 2016; 11: 839 872, doi: 10.2147/COPD.S49378, indexed in Pubmed: 27143874.

14. Hawkins NM, Petrie MC, Jhund PS, et al. Heart failure and chronic obstructive pulmonary disease: diagnostic pitfalls and epidemiology. Eur J Heart Fail. 2009; 11(2): 130-139, doi: 10.1093/eurjhf/hfn013, indexed in Pubmed: 19168510.

15. Liu Y, Yan S, Poh K, et al. Impact of air quality guidelines on COPD sufferers. Int J Chron Obstruct Pulmon Dis. 2016; 11: 839-872, doi: 10.2147/COPD.S49378, indexed in Pubmed: 27143874

16. Wu S, Ni Y, Li H, et al. Short-term exposure to high ambient air pollution increases airway inflammation and respiratory symptoms in chronic obstructive pulmonary disease patients in Beijing, China. Environ Int. 2016; 94: 76-82, doi: 10.1016/j. envint.2016.05.004, indexed in Pubmed: 27209003.

17. Karakatsani A, Analitis A, Perifanou D, et al. Particulate matter air pollution and respiratory symptoms in individuals having either asthma or chronic obstructive pulmonary disease: a European multicentre panel study. Environ Health. 2012; 11: 75, doi: 10.1186/1476-069X-11-75, indexed in Pubmed: 23039312.

18. DeVries R, Kriebel D, Sama S. Low level air pollution and exacerbation of existing copd: a case crossover analysis. Environ Health. 2016; 15(1): 98, doi: 10.1186/s12940-016-0179-z, indexed in Pubmed: 27756407.

19. Moore E, Chatzidiakou L, Kuku MO, et al. Global Associations between Air Pollutants and Chronic Obstructive Pulmonary Disease Hospitalizations. A Systematic Review. Ann Am Thorac Soc. 2016; 13(10): 1814-1827, doi: 10.1513/AnnalsATS. 201601-064OC, indexed in Pubmed: 27314857. 
20. DeVries R, Kriebel D, Sama S. Outdoor Air Pollution and COPD-Related Emergency Department Visits, Hospital Admissions, and Mortality: A Meta-Analysis. COPD. 2017; 14(1): 113-121, doi: 10.1080/15412555.2016.1216956, indexed in Pubmed: 27564008.

21. Aga E, Samoli E, Touloumi G, et al. Confounding and effect modification in the short-term effects of ambient particles on total mortality: results from 29 European cities within the APHEA2 project. Epidemiology. 2001; 12(5): 521-531, indexed in Pubmed: 11505171.

22. Laden F, Neas LM, Dockery DW, et al. Association of fine particulate matter from different sources with daily mortality in six U.S. cities. Environ Health Perspect. 2000; 108(10): 941-947, doi: 10.1289/ehp.00108941, indexed in Pubmed: 11049813.

23. Bell ML, Ebisu K, Peng RD, et al. Hospital admissions and chemical composition of fine particle air pollution. Am J Respir Crit Care Med. 2009; 179(12): 1115-1120, doi: 10.1164/rccm. 200808-1240OC, indexed in Pubmed: 19299499.

24. Casas L, Simons K, Nawrot TS, et al. Respiratory medication sales and urban air pollution in Brussels (2005 to 2011). Environ Int. 2016; 94: 576-582, doi: 10.1016/j.envint.2016.06.019, indexed in Pubmed: 27346740.

25. Magzamen S, Oron AP, Locke ER, et al. Association of ambient pollution with inhaler use among patients with COPD: a panel study. Occup Environ Med. 2018; 75(5): 382-388, doi: 10.1136/ oemed-2017-104808, indexed in Pubmed: 29535158.

26. Li R, Jiang N, Liu Q, et al. Impact of Air Pollutants on Outpatient Visits for Acute Respiratory Outcomes. Int J Environ Res Public Health. 2017; 14(1), doi: 10.3390/ijerph14010047, indexed in Pubmed: 28067786.

27. Wang KY, Chau TT. An association between air pollution and daily outpatient visits for respiratory disease in a heavy industry area. PLoS One. 2013; 8(10): e75220, doi: 10.1371/journal. pone.0075220, indexed in Pubmed: 24204573.

28. McCormack MC, Belli AJ, Waugh D, et al. Respiratory Effects of Indoor Heat and the Interaction with Air Pollution in Chronic Obstructive Pulmonary Disease. Ann Am Thorac Soc. 2016; 13(12): 2125-2131, doi: 10.1513/AnnalsATS.201605-329OC, indexed in Pubmed: 27684429.

29. Bernstein AS, Rice MB. Lungs in a warming world: climate change and respiratory health. Chest. 2013; 143(5): 1455-1459, doi: 10.1378/chest.12-2384, indexed in Pubmed: 23648909.
30. Liang WM, Liu WP, Kuo HW. Diurnal temperature range and emergency room admissions for chronic obstructive pulmonary disease in Taiwan. Int J Biometeorol. 2009; 53(1): 17-23, doi: 10.1007/s00484-008-0187-y, indexed in Pubmed: 18989710.

31. Ferrari U, Exner T, Wanka ER, et al. Influence of air pressure, humidity, solar radiation, temperature, and wind speed on ambulatory visits due to chronic obstructive pulmonary disease in Bavaria, Germany. Int J Biometeorol. 2012; 56(1): 137-143, doi: 10.1007/s00484-011-0405-x, indexed in Pubmed: 21301889.

32. Almagro P, Hernandez C, Martinez-Cambor P, et al. Seasonality, ambient temperatures and hospitalizations for acute exacerbation of COPD: a population-based study in a metropolitan area. Int J Chron Obstruct Pulmon Dis. 2015; 10: 899-908, doi: 10.2147/COPD.S75710, indexed in Pubmed: 26056439.

33. Chen Y, Zang Lu, Du W, et al. Ambient air pollution of particles and gas pollutants, and the predicted health risks from longterm exposure to PM in Zhejiang province, China. Environ Sci Pollut Res Int. 2018 [Epub ahead of print], doi: 10.1007/ s11356-018-2420-5, indexed in Pubmed: 29876857.

34. Wen CP, Gao W. PM: an important cause for chronic obstructive pulmonary disease? Lancet Planet Health. 2018; 2(3): e105-e106, doi: 10.1016/S2542-5196(18)30025-1, indexed in Pubmed: 29615221

35. Rovira J, Sierra J, Nadal M, et al. Main components of PM in an area influenced by a cement plant in Catalonia, Spain: Seasonal and daily variations. Environ Res. 2018; 165: 201-209, doi: 10.1016/j.envres.2018.04.010, indexed in Pubmed: 29727820.

36. Sinharay R, Gong J, Barratt B, et al. Respiratory and cardiovascular responses to walking down a traffic-polluted road compared with walking in a traffic-free area in participants aged 60 years and older with chronic lung or heart disease and age-matched healthy controls: a randomised, crossover study. Lancet. 2018; 391(10118): 339-349, doi: 10.1016/S01406736(17)32643-0, indexed in Pubmed: 29221643.

37. Liu Yi, Sun J, Gou Y, et al. A Multicity Analysis of the Short-Term Effects of Air Pollution on the Chronic Obstructive Pulmonary Disease Hospital Admissions in Shandong, China. Int J Environ Res Public Health. 2018; 15(4), doi: 10.3390/ ijerph15040774, indexed in Pubmed: 29673181.

38. Bloemsma LD, Hoek G, Smit LAM. Panel studies of air pollution in patients with COPD: Systematic review and meta-analysis. Environ Res. 2016; 151: 458-468, doi: 10.1016/j. envres.2016.08.018, indexed in Pubmed: 27565881. 